\title{
DIRECTION OF ARRIVAL ESTIMATION USING MUSIC ALGORITHM
}

\author{
Revati Joshi ${ }^{1}$, Ashwinikumar Dhande ${ }^{2}$ \\ ${ }^{I}$ Student, E\&Tc Department, Pune Institute of Computer Technology, Maharashtra, India \\ ${ }^{2}$ Professor, E\&Tc Department, Pune Institute of Computer Technology, Maharashtra, India
}

\begin{abstract}
The performance of smart antenna greatly depends on the effectiveness of DOA estimation algorithm. This paper analyzed the performance of MUSIC (Multiple Signal Classification) algorithm for DOA estimation. simulation results shows that MUSIC provide better angular resolution for increasing number of array element, distance between array element and number of samples. All the simulations are carried out using MATLAB.
\end{abstract}

Keywords: DOA (Direction of arrival), MUSIC (Multiple signal classification), ULA(Uniform linear array)

\section{INTRODUCTION}

The technique used for estimating the direction of arrival(DOA) of radio signals in wireless system has received considerable attention because estimating the direction of arrival of several radio signals impinging on an array of sensors is required in a variety of other applications, including radar, sonar and seismology[1-2].

DOA estimation using a fixed antenna has many disadvantages such as its resolution is limited by antenna's main lobe beam width [3].Antenna's mainlobe beam width is inversely proportional to physical size. Thus improving accuracy of angle measurement by increasing physical size of receiving antenna is not always a practical solution. certain systems such as a missile seeker or aircraft antenna have physical size limitation, therefore they provide wide main lobe beam width. So that resolution is poor.

Instead of using a single antenna ,an array antenna system with innovative signal processing can enhance the resolution of signal DOA[4]. A array antenna has better performance in signal reception and parameter estimation.

As shown in fig.1 DOA estimation is depends on many parameters such as number of mobile users, inter element spacing, number of signals and spatial distribution.

There are many different super resolution algorithms such as spectral estimation, model based and Eigen analysis [5]. In this paper we concentrate on estimation of DOA using MUSIC (Multiple signal classification) algorithm.
$* * *$

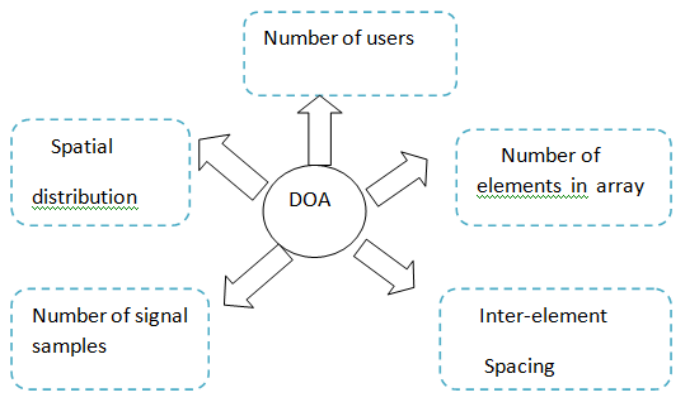

Fig-1:.DOA Estimation Parameter.

\section{ARRAY SENSOR SYSTEM}

The system consists of ULA with M antenna elements that are linearly spaced along with equal distance.

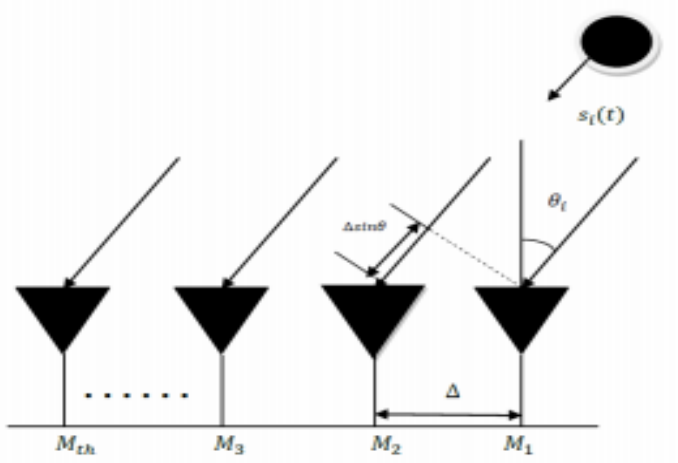

Fig.2: System model for DOA estimation using a uniform linear array of $M$ elements.

The M elements of ULA receives signals from a remote souce i. The spacing between the elements $\Delta$ should be half wavelength of received signals. Suppose a plane wave signal 
generated by source $\mathrm{i}$ impinging on the array at an angle $\theta_{i}$ and the signal generated by the source $\mathrm{i}$ is the $s_{i}(t)$.If all the signals generated by all the D sources, $s_{i}(t), 1<\mathrm{i}<\mathrm{D}$, then total signal and noise received by mth array element is given in $\mathrm{eq}^{\mathrm{n}} 1$.

$$
x_{m}(t)=s_{i}(t) \sum_{i=1}^{D} e^{j(m-1) \varphi_{i+n_{m}}}(t), \mathrm{m}=1
$$

The equation 1 can be written as

$$
\begin{aligned}
& x_{m}(t)=\left[\begin{array}{ll}
\mathrm{a}\left(\varphi_{o}\right. & a\left(\varphi_{1}\right) \ldots a\left(\varphi_{D-1}\right)
\end{array}\right]\left[\begin{array}{c}
s_{0}(t) \\
s_{1}(t) \\
s_{D-1}(t)
\end{array}\right]+\mathrm{n}(\mathrm{t}) \\
& =\mathrm{As}(\mathrm{t})+\mathrm{n}(\mathrm{t})
\end{aligned}
$$

Where,

The data column vector received by the array is given in equation 3.

$$
x(t)=\left[x_{1}(t), x_{1}(t), x_{m}(t)\right]^{T}
$$

The signal column vector generated by the sources is given in equation 4.

$$
s(t)=\left[s_{1}(t), s_{2}(t), s_{M}(t)\right]^{T}
$$

The noise vector generated by the sources is given in equation 5.

$$
n(t)=\left[n_{1}(t), n_{2}(t), n_{M}(t)\right]^{T}
$$

These noise vector is having zero mean spatially uncorrelated additive white noise and variance is equal to $\sigma_{N}^{2}$.

The array steering column vector $\mathrm{a}\left(\varphi_{i}\right)$ is defined in equation 6.

$$
a\left(\varphi_{i}\right)=\left[1, e^{j \varphi_{i}}, e^{j 2 \varphi_{i}}, e^{j(M-1) \varphi_{i}}\right]^{T}
$$

The steering matrix is given in equation 7 .

$$
\mathrm{A}=\left[\mathrm { a } \left(\varphi_{1}, \ldots \ldots a\left(\varphi_{i}\right) . . a\left(\varphi_{d}\right)\right.\right.
$$

Where $\varphi_{i}=-\frac{2 \pi}{\lambda} \Delta \sin \theta_{i}$.is called spatial frequency for the ith source that generates the signals of incident angle[6].

\section{MUSIC ALGORITHM}

MUSIC is an acronym stands for Multiple Signal Classification [7-8].It is proposed by Schmidt in 1979.The basic idea behind the MUSIC algorithm is to separate the signal from noise by using orthogonality property of their spaces through eigen decomposition of the correlation matrix of the received signal. It provides information about number of incident signals, direction of arrival (DOA) of each signal strengths and cross correlation between incident signals and noise power. It is assumed that noise in each channel is uncorrelated. This leads to a diagonal covariance matrix.

The Algorithm starts with initializing some parameters such as 1. Number of antenna element=M.

2. Spacing between antenna element $=\Delta$.

3. Number of sources $=s_{1}, s_{2} \ldots . s_{D}$.

4. Incoming signal direction $=\varphi_{1}, \varphi_{2} \ldots \ldots \varphi_{D}$.

5. Number of samples $=K$.

Using above data model, the input covariance matrix is given in equation 8 .

$$
R_{X X}=E\left[X(t) X(t)^{H}\right]
$$

Where $\mathrm{E}[$.$] is the expectation and (.)^{H}$ is hermitian conjugate transpose.

Substituting the value of eq. 2 in eq. 8 resultant expression for $R_{X X}$ can be obtain using eq. 9

$$
R_{X X}=A P A^{H}+\sigma^{2} I
$$

Where $\mathrm{P}$ is the covariance matrix of the signal vector is given in eq. 10

$$
P=E\left[s(t) s(t)^{H}\right]
$$

$\sigma^{2}$ is the noise covariance matrix is given in eq.11

$$
E\left[n(t) n(t)^{H}\right]
$$

I is $\mathrm{M}^{*} \mathrm{M}$ identity matrix.

By analyzing the properties of $R_{X X}$ if numbers of antenna elements are greater than the number of sources, then $\mathrm{P}$ is positive definite i.e signal are not fully correlated.

The array covariance matrix can be estimated from received snapshot vectors can be obtain using eq.12

$$
\widehat{R_{X X}}=\frac{1}{K} \sum_{K=1}^{K} X(t) X(t)^{H}
$$

Where $\mathrm{K}$ is the number of snapshot

Perform eigen decomposition of spatial correlation matrix If $\left(\lambda_{0}, \lambda_{1}, \ldots . \lambda_{M-1}\right)$ are eigen values of $R_{X X}$.

Solving for eigenvalue of $R_{X X}$ is given in eq.13,14,15.

$$
\left|R_{X X}-\lambda_{i} I\right|=0
$$




$$
\begin{aligned}
& \left|A P A^{H}+\sigma^{2}-\lambda_{i}\right|=0 \\
& \left|A P A^{H}+\left(\sigma^{2}-\lambda_{i}\right)\right|=0
\end{aligned}
$$

Therefore eigen values $v_{i}$ of $A P A^{H}$ are obtain using eq.16

$$
v_{i}=\sigma^{2}-\lambda_{i}
$$

As A contains steering vectors which are independent and full column rank.and $\mathrm{p}$ is positive definite. Therefore eigenvalue $v_{i}$ of $\mathrm{AP} A^{H}$ are zero.M-D eigenvalue of $R_{X X}$ are equal to the noise variance $\sigma^{2}$.sort the Eigen values of $R_{X X}$ such that $\lambda_{0}$ is the largest Eigen value, and $\lambda_{M-1}$ is the smallest Eigen value. Therefore,

$$
\begin{aligned}
& \lambda_{D}, \lambda_{1 \ldots \ldots \ldots \ldots . . .} \lambda_{M-1}=\sigma^{2} \\
& \widehat{D}=M-K
\end{aligned}
$$

The Eigen vector associated with a particular Eigen value, $\lambda_{i}$ is the vector $q_{i}$ such as given in eq.19

$$
R_{X X}-\lambda_{i} I=O
$$

For Eigen vectors associated with M-D smallest Eigen values, obtain using eq.20,21.

$$
\begin{gathered}
\left(R_{X X}-\sigma_{n}^{2} I\right) q_{i=} A P A^{H} \mathrm{q}_{\mathrm{i}}+\sigma_{n}^{2} I-\sigma^{2} I=0 \\
A P A^{H} \mathrm{q}_{\mathrm{i}}=0
\end{gathered}
$$

As $\mathrm{A}$ is full rank and $\mathrm{P}$ is non-singular, so that eq. 21 becomes

$$
A^{H} \mathrm{q}_{\mathrm{i}}=0
$$

This means that the Eigen vectors associated with the M-D smallest Eigen values are orthogonal to the D steering vectors that make up A.

$$
\left\{\mathrm{a}\left(\varphi_{0}\right) \ldots \ldots \ldots a\left(\varphi_{D-1}\right)\right\} \downarrow\left\{q_{D}, \ldots \ldots . . q_{M-1}\right\}
$$

To search the noise subspace, we form a matrix containing the noise Eigen vectors.

$$
V_{n}=\left[\begin{array}{lllll}
q_{D} & q_{D+1} & \ldots & \ldots & \ldots \\
q_{M-1}
\end{array}\right]
$$

Since the steering vectors corresponding to signal components are orthogonal to the noise subspace Eigen vectors, $a^{H}(\varphi) V_{n} V_{n}^{H} \mathrm{a}(\varphi)=0$ for $\varphi$ corresponding to the DOA of a multipath component. Then the DOAs of the multiple incident signals can be estimated by locating the peaks of a MUSIC spatial spectrum which is given by eq. 24

$$
P_{\text {Music }}(\varphi)=\frac{1}{a^{H}(\varphi) V_{n} V_{n}^{H} \mathrm{a}(\varphi)}
$$

\section{SIMULATION RESULT}

The performance of MUSIC algorithm depends on following parameters

1. Number of snapshots.

2. Number of array elements.

3. Element separation .

\subsection{Effect of the Number of Snapshots on MUSIC} Algorithm.

As shown in Fig.(3) as number of snapshots $(\mathrm{k}=1000)$ increases, MUSIC can accurately estimate the DOA of incident signals. This is because increase the number of snapshots, will make narrower beam width around incident signals directions. As shown in fig.(4) if number of snapshot $(\mathrm{k}=10)$ then resolution of Music algorithm decreases.

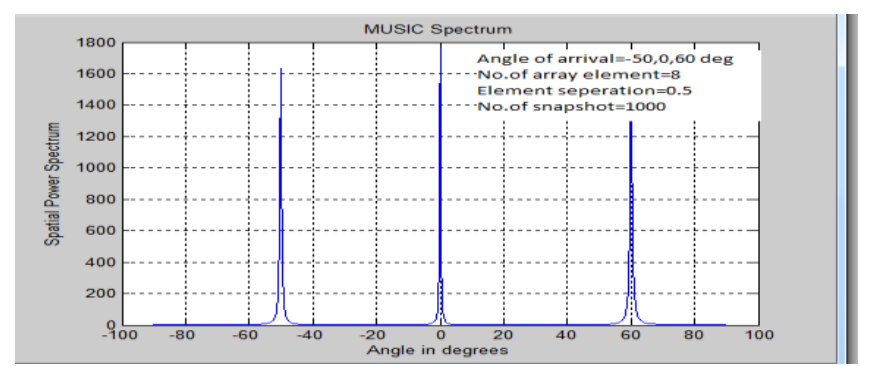

Fig-3: MUSIC Spectrum using no of snapshot $=1000$

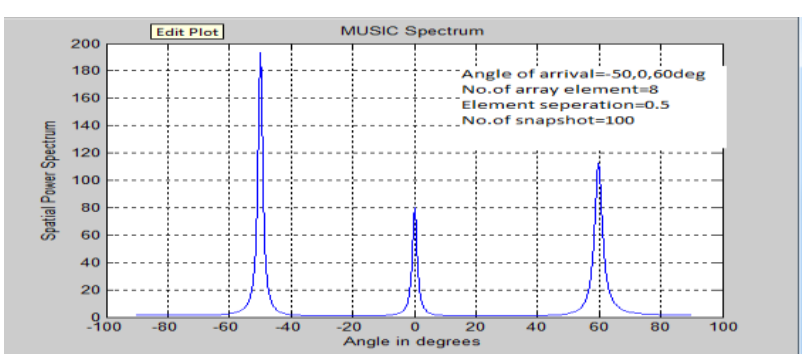

Fig-4: MUSIC Spectrum using no of snapshot=100.

\subsection{Effect of the Number of Array Elements on MUSIC Algorithm}

As shown in Fig. (5) it is clear, that as number of array elements increases, MUSIC can accurately estimate the DOA of incident signals. And if number of array element $(\mathrm{N}=4)$ decreases, then resolution of Music algorithm decreases. It is shown in Fig (6). 


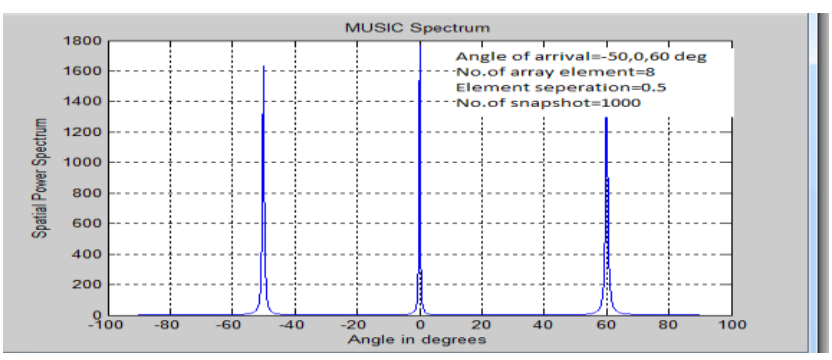

Fig-.5: MUSIC Spectrum using no of array element=8.

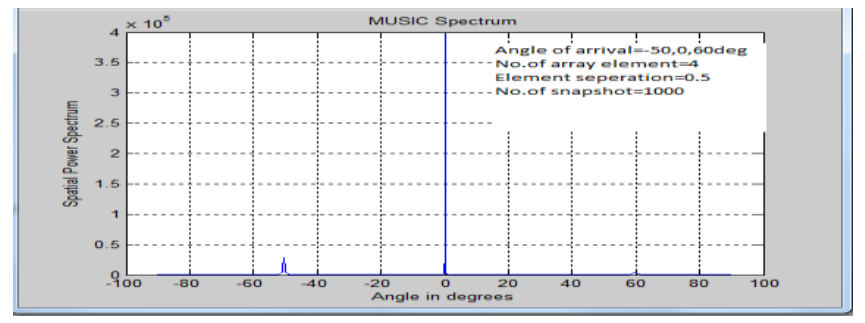

Fig-6::MUSIC Spectrum using no of array element=4.

\subsection{Effect of the Varying Element Separation on MUSIC Algorithm}

From Fig. (7) it is clear, that as the distance between array elements increases, MUSIC can accurately estimate the DOA of incident signals. And fig.(8) shows that, if distance between array element is $(\mathrm{d}=0.4)$ decreases, then resolution of Music algorithm is decreases.

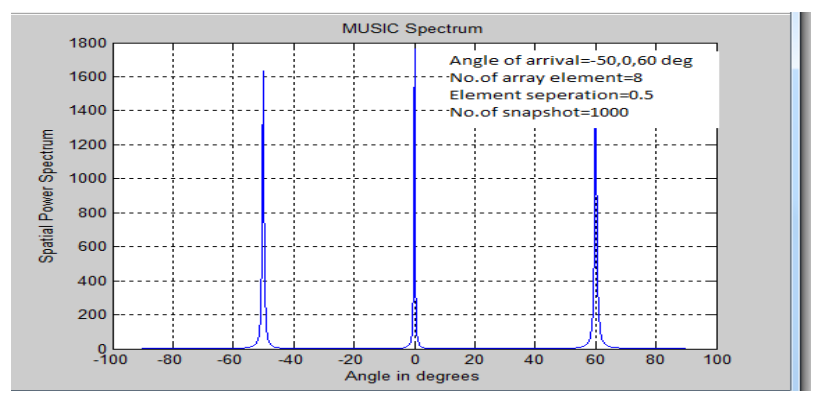

Fig-7: MUSIC Spectrum using element separation $\mathrm{d}=0.5$.

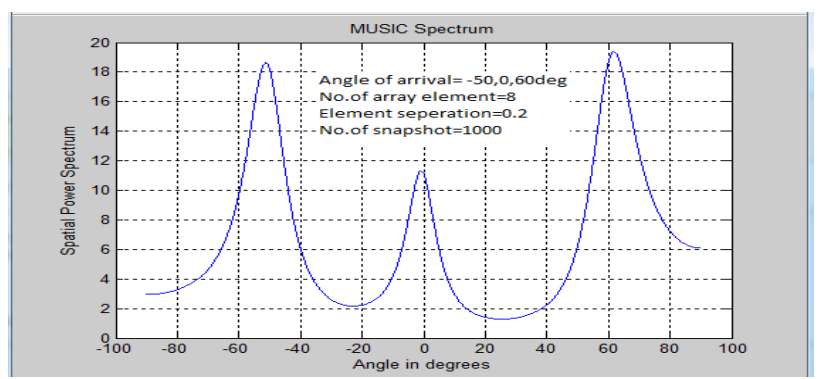

Fig-8: MUSIC Spectrum using element separation $\mathrm{d}=0.5$.

\section{CONCLUSIONS}

The simulation result of Music algorithm shows that angular resolution of Music algorithm improves with more no. of elements in the array, with large snapshot of signals and greater angular separation between the signals. MUSIC can estimate uncorrelated signal very well but it fails to detect correlated signals.

\section{REFERENCES}

[1]. S.U.Pillai, Array Signal Processing, Springer-Verlag, 1989.

[2]. M. Brandstein and D. Ward, Eds., Microphone Arrays, Springer, 2001.

[3]. Marshall M.Grice, Direction of arrival estimation using superresolution algorithms.M Sc. Thesis, California State Polytechnic University, Pomona, 2007.

[4]. Zekeriya Aliyazicioglu, H.K. Hwang. Marshall Grice, AnatolyYakovlev, "Sensitivity analysis for direction of arrival estimation using a Root-MUSIC algorithm," Proceedings of the International MultiConference of Engineers and Computer Scientists Vol II IMECS, 19-21 March 2008

[5]. Don H. Johnson, Dan E. Dudgeon, "Array signal processing concepts and techniques," P T R Prentice Hall Inc, A Division of Simon \& Schuster Englewood Cliffs, New Jersey 07632, 1993.

[6]. Z. Chen, G. Gokeda, and Y. Yu, "Introduction to Direction-of-Arrival Estimation", Artech House, vol. 34, no. 37, pp. 46-47, 2010

[7]. MUSIC Algorithm by Brian L. Evans, 211-105 Cory Hall, Berkeley, CA 94720-1772.

[8]. A.Majdoubi., M.Essaaidi, "The Estimation of DOA in Smart Antenna System", International Journal of Innovative Technology and Exploring Engineering, Vol.1, Issue6, Nov.2013. 